\title{
Superficial siderosis of the central nervous system associated with incomplete dural closure following posterior fossa surgery: report of 3 cases
}

\author{
Ravi Kumar, MD, ${ }^{1}$ Jeffrey T. Jacob, MD, ${ }^{1}$ Kirk M. Welker, MD, ${ }^{2}$ Fred M. Cutrer, MD, ${ }^{3}$ \\ Michael J. Link, MD, ${ }^{1}$ John L. D. Atkinson, MD, ${ }^{1}$ and Nicholas M. Wetjen, MD${ }^{1}$ \\ Departments of ${ }^{1}$ Neurosurgery, ${ }^{2}$ Radiology, and ${ }^{3}$ Neurology, Mayo Clinic, Rochester, Minnesota
}

This report reviews a series of 3 patients who developed superficial siderosis following posterior fossa operations in which dural closure was incomplete. In all 3 patients, revision surgery and complete duraplasty was performed to halt the progression of superficial siderosis. Following surgery, 2 patients experienced resolution of their CSF xanthochromia while 1 patient had reduced CSF xanthochromia. In this paper the authors also review the etiology, pathophysiology, diagnosis, and treatment of this condition. The authors suggest that posterior fossa dural patency and pseudomeningocele are risk factors for the latent development of superficial siderosis and recommend that revision duraplasty be performed in patients with posterior fossa pseudomeningoceles and superficial siderosis to prevent progression of the disease.

http://thejns.org/doi/abs/10.3171/2014.12.JNS141920

KEY WORDS superficial siderosis; posterior fossa; sensorineural hearing loss; pseudomeningocele

$\mathrm{S}$ UPERFICIAL siderosis is a syndrome notable for deposition of hemosiderin in the subpial and subependymal margins of the CNS. It is associated with sensorineural hearing loss, cerebellar ataxia, dementia, and myelopathy. ${ }^{5}$ The underlying cause of superficial siderosis is believed to be chronic subarachnoid hemorrhage ${ }^{4,7,15}$ caused by a number of entities including frank subarachnoid bleeding, dural pathologies, vascular tumors, and vascular abnormalities..$^{5}$ In this report we present 3 cases of superficial siderosis following incomplete closure of the dura after operations on the posterior fossa. We will discuss the pathogenesis and radiological findings of superficial siderosis, as well as our recommendations for the prevention of superficial siderosis in patients who must undergo posterior fossa surgery.

\section{Methods}

Institutional review board approval for the study was obtained from the Mayo Clinic. A retrospective review of all patients at our institution with diagnosis codes for posterior fossa pseudomeningoceles and superficial siderosis from 2004 to 2014 revealed 3 patients. A review of the literature was performed by searching PubMed with the key word/term "superficial siderosis."

\section{Case Reports}

Case 1

History and Examination

A 16-year-old girl presented to her neurologist with a 
complaint of chronic headaches associated with nausea, photophobia, and phonophobia. Her history was remarkable for partial trisomy 8 due to a ringed chromosome 8 , epilepsy, and history of a Chiari Type I malformation with associated syringomyelia treated by surgical decompression. Her Chiari decompression was performed 4 years earlier, and notably the dura was not closed. Following the procedure, she developed an external CSF leak through the surgical incision and ultimately required a right ventriculoperitoneal (VP) shunt. CSF shunting resolved the CSF leak and serial follow-up MRI and CT examinations of her head showed the absence of ventriculomegaly. Of note, the patient's mother reported that the patient had progressive hearing loss and ataxia that was not present prior to her Chiari decompression. A subsequent audiogram revealed bilateral sensorineural hearing loss with a pure tone average (PTA) of $27.5 \mathrm{~dB}$ and a word recognition score (WRS) of $100 \%$ at $40 \mathrm{~dB}$ bilaterally.

Subsequent MRI of her head showed development of magnetic susceptibility, consistent with hemosiderin deposition along the folia of the cerebellum, around the brainstem, and in the bilateral occipital sulci, suggestive of superficial siderosis (Fig. 1A). The patient also had a stable occipital pseudomeningocele that had ossified (Fig. 1B). No ventriculomegaly was noted. A lumbar puncture was performed and demonstrated xanthochromia with a significant number of red blood cells (RBCs; $n=1078)$.

\section{Operation and Postoperative Course}

Given the patient's radiological findings and clinical picture, superficial siderosis was the most likely explanation for the patient's symptoms. The patient was taken to the operating room for revision of her wound and duraplasty using bovine pericardium. She tolerated the procedure well and was discharged home on postoperative Day 5. A repeat lumbar puncture (on postoperative Day 45) revealed resolution of xanthochromia and no RBCs. She has remained neurologically stable with no further decline in hearing or increasing ataxia.

\section{Case 2}

History and Presentation

A 36-year-old man presented to our institution with bilateral hearing loss and ataxia. At age 15 he acquired meningitis that resulted in hydrocephalus and the eventual need for bilateral VP shunts. At the age of 32, he underwent decompression of a Chiari Type I malformation at an outside hospital. His presenting symptom at that time was headache. For 6 months following his posterior fossa decompression, he experienced relief of his headaches. The operative report did not specify if a duraplasty or dural closure procedure was performed at the initial operation. His headaches recurred and he was taken back to the operating room at an outside institution for a dural patch graft because of a presumed pseudomeningocele. Following the patching of his dura, his headaches resolved. Two years after the duraplasty, an MRI examination revealed a stable pseudomeningocele and no evidence of superficial siderosis. Almost 5 years after his original surgery he presented to our institution with ataxia and sensorineural hearing
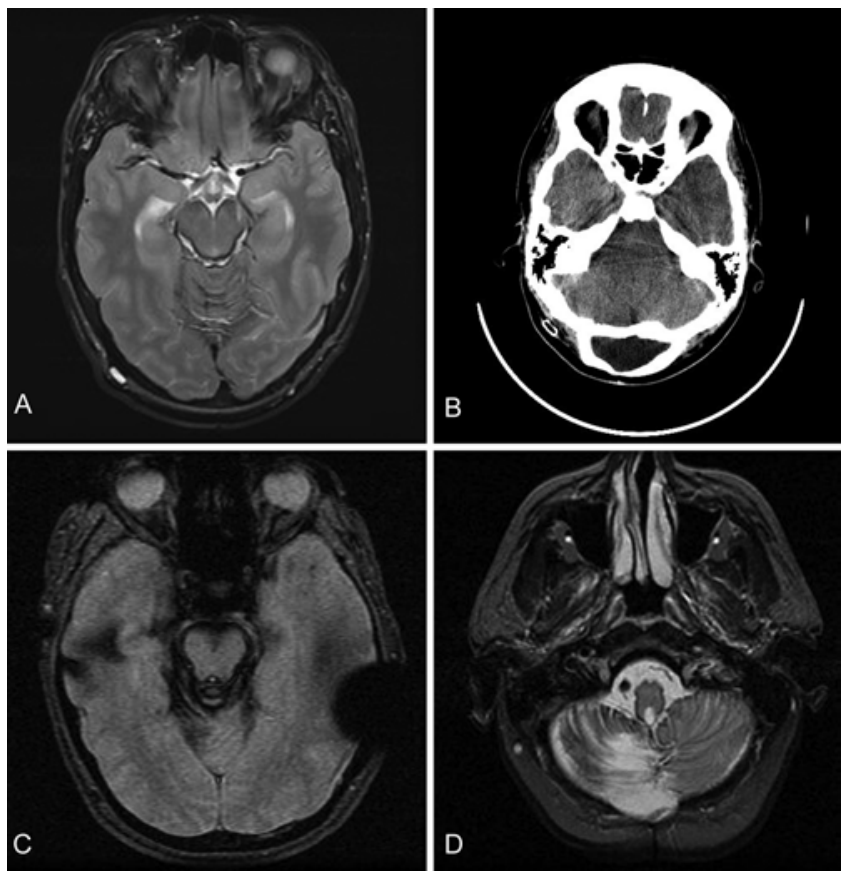

FIG. 1. Axial images of the 3 patients. A: Case 1. Conventional spin echo T2-weighted MR image showing a hypointense border around the midbrain due to magnetic susceptibility caused by hemosiderin deposition. There is similar T2 signal hypointensity along the cerebellar folia bilaterally. B: Case 1. Head CT scan displayed in a brain window demonstrating fluid attenuation in the ossified pseudomeningocele, consistent with CSF. C: Case 2. MR image of the head with a gradient echo sequence showing hemosiderin deposition in the pons and cerebellar folia. Note the susceptibility artifact over the left temporal lobe due to extracranial hardware. D: Case 3. T2-weighted MR image showing hemosiderin lining the medulla and cerebellar folia, and a midline pseudomeningocele.

loss with a PTA of $19 \mathrm{~dB}$ and a WRS of $100 \%$ at $40 \mathrm{~dB}$ bilaterally. An MR image revealed superficial siderosis extending from the midbrain down to the upper cervical cord (Fig. 1C). There was also a rim of siderosis lining the pseudomeningocele. A lumbar puncture was performed and was positive for xanthochromia, RBCs $(n=202)$, and iron-laden macrophages.

\section{Operation and Postoperative Course}

The patient was taken to surgery for autologous fascia lata duraplasty. The patient tolerated the surgery well and subsequent MR images showed resolution of his pseudomeningocele and excellent wound healing. Two subsequent lumbar punctures showed decreasing xanthochromia. The patient was lost to follow-up thereafter.

\section{Case 3}

History and Presentation

A 51-year-old man presented to our institution complaining of progressive bilateral hearing loss, unsteady gait, and visual loss. Twenty-six years prior, the patient had undergone resection of a posterior fossa mass, diagnosed as microglioma, followed by 5000 cGy of wholebrain radiation. An audiogram revealed bilateral sensori- 
neural hearing loss with a PTA of $32.5 \mathrm{~dB}$ and a WRS of $95 \%$ on the right and $40 \%$ on the left at $40 \mathrm{~dB}$. MRI of the brain revealed an enhancing mass surrounding the left optic nerve and supraclinoid carotid artery, extending onto the planum sphenoidale. Extensive superficial siderosis in the posterior fossa and a midline infratentorial pseudomeningocele were also revealed (Fig. 1D). A lumbar puncture demonstrated RBCs $(\mathrm{n}=307)$ and xanthochromia. A CT myelogram of the entire neuraxis was performed, confirming an area of CSF egress in the posterior fossa.

\section{Operation and Postoperative Course}

The patient was taken to the operating room for repair of this posterior fossa dura using autologous fascia lata. A lumbar puncture performed 1 year later showed minimal RBCs $(n=2)$ and the absence of xanthochromia. The patient remains neurologically stable.

\section{Discussion}

Superficial siderosis is a rare and irreversible disease marked by hemosiderin deposition in the subpial and subependymal tissues of the CNS. ${ }^{5}$ The disease leads to sensorineural hearing loss, cerebellar ataxia, dementia, and myelopathy. Blood in the subarachnoid space is the most common identifiable cause, although a bleeding source cannot always be identified. ${ }^{23}$ The etiologies of subarachnoid blood leading to superficial siderosis are somewhat broad and heterogeneous, and include tumors, ${ }^{1,13,20}$ vascular malformations, ${ }^{7}$ brachial plexus injuries, ${ }^{4}$ nerve root avulsions, ${ }^{2}$ cerebral amyloid angiopathy, ${ }^{25}$ and iatrogenic, traumatic, and pathological dural defects. ${ }^{14,18}$ A study of 1062 patients in the Netherlands found an overall prevalence of $0.7 \%$ in the general population, and all of these cases were associated with cerebral microbleeds. ${ }^{25}$

Five steps have been identified in the pathophysiology of superficial siderosis: 1) chronic or intermittent subarachnoid hemorrhage; 2) hemolysis of RBCs; 3) entry of heme into CNS tissue; 4) conversion of heme to hemosiderin; and 5) resulting oxidative damage to the affected cells. ${ }^{12}$ Hemeoxygenase- 1 is a key enzyme in the conversion of heme to hemosiderin and is only transiently activated in response to single-event subarachnoid hemorrhages. ${ }^{11}$ This may explain the lack of association between acute subarachnoid hemorrhage and superficial siderosis. The CNS has a very limited ability to expel iron, thus accumulation of iron is inevitable when chronic and intermittent bleeding into the subarachnoid space occurs. ${ }^{12}$ Both heme and iron damage the CNS by way of oxidation and the creation of free radicals. The cerebellar cortex and cranial nerve VIII tend to be the most susceptible areas of the CNS to develop superficial siderosis. The Bergman glia and microglia of the cerebellar cortex act as conduits for the entrance of heme into the tissues and for ferritin biosynthesis. ${ }^{10,12}$ Cranial nerve VIII transitions from CNS tissue to peripheral nervous system tissue at the internal acoustic meatus, unlike the other cranial nerves that transition at the brainstem. This makes cranial nerve VIII exquisitely sensitive to iron deposition due to the presence of Bergman glia in its cisternal segment. ${ }^{10}$ Staining of tissues affected by superficial siderosis will show iron accumula- tion within macrophages, superficial astrocytes, and gray matter oligodendroglia. ${ }^{9}$

The link between CSF hypotension due to high- and low-flow dural defects and superficial siderosis has been thoroughly noted in the literature..$^{18}$ One study found that of 22 patients with superficial siderosis, 15 had a history of trauma and 14 had fluid-filled spaces on spinal MRI suggesting dural defects. ${ }^{16}$ Often a patient with superficial siderosis will have overlapping symptoms with CSF hypotension, including orthostatic headache, imbalance, and back pain. These patients warrant craniospinal imaging as many of them will have spinal dural defects that may be surgically repairable. ${ }^{19}$

The advent of MRI has led to an increase in the diagnosis of superficial siderosis. T2-weighted MRI in superficial siderosis shows a rim of hypointensity surrounding affected tissues (Fig. 1A, C, and D). ${ }^{15}$ Gradient recalled echo and susceptibility-weighted imaging sequences can provide even greater sensitivity for superficial siderosis compared with traditional conventional spin echo and fast spin echo T2-weighted images. ${ }^{26}$ The cerebellum, brainstem, and cisternal segments of the cranial nerves are the most commonly involved areas, although superficial siderosis can be visualized around the major fissures and cortical sulci. Cerebellar atrophy can also be observed. ${ }^{15}$

Imaging in superficial siderosis is not only important in establishing the diagnosis but also in identification of the source of bleeding. Dural defects are often the source of chronic subarachnoid bleeds. ${ }^{18}$ Dynamic CT myelography has been shown to be effective in locating a connection between epidural cystic fluid collections and the intrathecal space. ${ }^{17}$ Digital subtraction myelography can be effective in localizing a dural tear in a patient with superficial siderosis when myelography, postmyelography CT, or dynamic CT myelography has failed. ${ }^{6}$ Rapid extravasation of contrast material can quickly pinpoint the dural defect and help with preoperative planning. Friable vessels at the borders of dural tears could be the source of chronic subarachnoid hemorrhages leading to superficial siderosis, indicating surgical correction as an available solution. Cerebral and spinal angiography are generally low-yield diagnostic tests in the setting of superficial siderosis, as the bleeding source is often slow and intermittent. ${ }^{15}$

In all our cases, the patients had an obvious dural defect from previous posterior fossa operations with incomplete dural closure. The first patient had a large chronic pseudomeningocele that had ossified over time (Fig. 1B). The presence of posterior fossa fluid collections has been reported in association with superficial siderosis. ${ }^{3,8}$ All patients' lumbar punctures revealed xanthochromia and RBCs, giving evidence of active and, most likely, chronic subarachnoid bleeding. Extradural fluid collections when in continuity with the intradural space allow for decreased CSF pressure by shunting fluid into the surrounding tissue. In addition, 2 patients (Cases 1 and 2) had VP shunts, potentially contributing to a decrease in CSF pressure. The pathogenesis of superficial siderosis in association with CSF hypovolemia and a dural defect has been postulated to result from dural tears containing friable vessels that bleed into the subarachnoid space and/or extravasation of RBCs through engorged vessels due to a low-pressure environment. ${ }^{18}$ 
Surgeries in the posterior fossa have been reported to lead to superficial siderosis. In 1 reported case, a pontine cavernous malformation was excised, leaving a fistulous connection with the fourth ventricle and allowing for chronic subarachnoid bleeding and the development of superficial siderosis. ${ }^{14}$ Another small patient series cited the development of superficial siderosis after resection of cerebellar tumors ( 3 astrocytomas and 1 medulloblastoma) in 4 pediatric patients. ${ }^{1}$ The authors postulated that the large cystic cavities that were left after surgery could have created multiple bleeding points with access to the ventricles. The report did not indicate whether watertight dural closure had been performed in any of the cases.

Superficial siderosis is a disease of delayed onset. Multiple reports have noted inciting conditions in children that did not present with symptoms of superficial siderosis until years later. ${ }^{1,4,21}$ These studies strongly suggest that chronic, slow, subarachnoid bleeds are required to develop superficial siderosis. This is also evidenced by the fact that patients with acute subarachnoid hemorrhages, such as from aneurysm rupture, do not develop superficial siderosis. In 1 case report, a patient who underwent resection of an astrocytoma from the cerebellar vermis developed symptomatic superficial siderosis 29 years later. ${ }^{22}$ The longest reported delay in onset of symptoms has been 37 years, demonstrating the chronicity of the disease and a large potential window for intervention..$^{21}$ The 3 patients in the current report developed symptoms severe enough to warrant investigation 4,5, and 26 years, respectively, after the initial intervention.

To our knowledge, only 1 other case of superficial siderosis after Chiari malformation decompression has been reported. ${ }^{21}$ In this case report, the authors postulated that the patient's superficial siderosis was due to chronic subdural hematomas from repeated shunt revisions. They did not mention whether the dura had been closed following Chiari decompression.

The etiology for superficial siderosis in all patients in this series was likely due to incomplete dural closure following posterior fossa surgery. The defect in the dura created a pseudomeningocele in all patients. Although speculative in nature, we postulate that the mechanism of development of superficial siderosis in these patients could have involved CSF hypotension due to the dural defect and VP shunt placement in Cases 1 and 2, and friable vessels in the open dural edges or in the pseudomeningocele cavity. Closure of the dura in Cases 1 and 3 led to resolution of xanthochromia and RBCs in the CSF, and in Case 2 led to a decreased degree of xanthochromia. Reoperation and dural closure was successful in eliminating the probable cause of subarachnoid bleeding. It is important to explain to patients that the damage done by superficial siderosis is likely irreversible. When a source of chronic subarachnoid bleeding can be identified, surgery is indicated to prevent development or progression of superficial siderosis. ${ }^{24}$

Superficial siderosis is a progressive disease that causes neurological deterioration associated with accumulation of iron in the subpial and subependymal tissues of the CNS. It may occur after posterior fossa surgery in which the dura was not closed. Patients with shunted hydrocephalus who undergo posterior fossa surgery may carry an increased risk of developing superficial siderosis in the latent postoperative period. Proper dural closure may play a role in reducing the risk of postoperative superficial siderosis, and revision duraplasty may reduce the progression of superficial siderosis in patients with posterior fossa pseudomeningoceles.

\section{References}

1. Anderson NE, Sheffield S, Hope JK: Superficial siderosis of the central nervous system: a late complication of cerebellar tumors. Neurology 52:163-169, 1999

2. Aquilina K, Kumar R, Lu J, Rawluk D: Superficial siderosis of the central nervous system following cervical nerve root avulsion: the importance of early diagnosis and surgery. Acta Neurochir (Wien) 147:291-297, 2005

3. Bürk K, Skalej M, Dichgans J: High prevalence of CSFcontaining cysts in superficial hemosiderosis of the central nervous system. J Neurol 248:1005-1006, 2001

4. Cohen-Gadol AA, Krauss WE, Spinner RJ: Delayed central nervous system superficial siderosis following brachial plexus avulsion injury. Report of three cases. Neurosurg Focus 16(5):E10, 2004

5. Fearnley JM, Stevens JM, Rudge P: Superficial siderosis of the central nervous system. Brain 118:1051-1066, 1995

6. Hoxworth JM, Patel AC, Bosch EP, Nelson KD: Localization of a rapid CSF leak with digital subtraction myelography. AJNR Am J Neuroradiol 30:516-519, 2009

7. Hsu WC, Loevner LA, Forman MS, Thaler ER: Superficial siderosis of the CNS associated with multiple cavernous malformations. AJNR Am J Neuroradiol 20:1245-1248, 1999

8. Jadhav TM, Hegde AU: Superficial siderosis: a rare occurrence in children. J Pediatr Neurosci 7:215-217, 2012

9. Kellermier H, Wang G, Wiley C: Iron localization in superficial siderosis of the central nervous system. Neuropathology 29:187-195, 2009

10. Koeppen AH, Dentinger MP: Brain hemosiderin and superficial siderosis of the central nervous system. J Neuropathol Exp Neurol 47:249-270, 1988

11. Koeppen AH, Dickson AC: Tin-protoporphyrin prevents experimental superficial siderosis in rabbits. J Neuropathol Exp Neurol 61:689-701, 2002

12. Koeppen AH, Michael SC, Li D, Chen Z, Cusack MJ, Gibson WM, et al: The pathology of superficial siderosis of the central nervous system. Acta Neuropathol 116:371-382, 2008

13. Konya D, Peker S, Ozgen S, Kurtkaya O, Necmettin Pamir M: Superficial siderosis due to papillary glioneuronal tumor. J Clin Neurosci 13:950-952, 2006

14. Kumar A, Aggarwal S, Willinsky R, TerBrugge KG: Posterior fossa surgery: an unusual cause of superficial siderosis. Neurosurgery 32:455-457, 1993

15. Kumar N: Neuroimaging in superficial siderosis: an in-depth look. AJNR Am J Neuroradiol 31:5-14, 2010

16. Kumar N, Cohen-Gadol AA, Wright RA, Miller GM, Piepgras DG, Ahlskog JE: Superficial siderosis. Neurology 66:1144-1152, 2006

17. Kumar N, Lindell EP, Wilden JA, Davis DH: Role of dynamic CT myelography in identifying the etiology of superficial siderosis. Neurology 65:486-488, 2005

18. Kumar N, McKeon A, Rabinstein AA, Kalina P, Ahlskog JE, Mokri B: Superficial siderosis and CSF hypovolemia: the defect (dural) in the link. Neurology 69:925-926, 2007

19. Kumar N, Miller GM, Piepgras DG, Mokri B: A unifying hypothesis for a patient with superficial siderosis, lowpressure headache, intraspinal cyst, back pain, and prominent vascularity. J Neurosurg 113:97-101, 2010

20. Matsumoto S, Kang Y, Sato S, Kawakami Y, Oda Y, Araki $\mathrm{M}$, et al: Spinal meningeal melanocytoma presenting with 
superficial siderosis of the central nervous system. Case report and review of the literature. J Neurosurg 88:890-894, 1998

21. McCarron MO, Flynn PA, Owens C, Wallace I, Mirakhur M, Gibson JM, et al: Superficial siderosis of the central nervous system many years after neurosurgical procedures. J Neurol Neurosurg Psychiatry 74:1326-1328, 2003

22. Miliaras G, Bostantjopoulou S, Argyropoulou M, Kyritsis A, Polyzoidis K: Superficial siderosis of the CNS: report of three cases and review of the literature. Clin Neurol Neurosurg 108:499-502, 2006

23. Offenbacher H, Fazekas F, Schmidt R, Kapeller P, Fazekas G: Superficial siderosis of the central nervous system: MRI findings and clinical significance. Neuroradiology 38 (Suppl 1):S51-S56, 1996

24. Shih P, Yang BP, Batjer HH, Liu JC: Surgical management of superficial siderosis. Spine J 9:e16-e19, 2009

25. Vernooij MW, Ikram MA, Hofman A, Krestin GP, Breteler MM, van der Lugt A: Superficial siderosis in the general population. Neurology 73:202-205, 2009

26. Wang J, Gong X: Superficial siderosis of the central nervous system: MR findings with susceptibility-weighted imaging. Clin Imaging 35:217-221, 2011

\section{Author Contributions}

Conception and design: Kumar, Jacob, Link, Atkinson, Wetjen. Acquisition of data: Kumar, Jacob, Link, Atkinson, Wetjen. Analysis and interpretation of data: Kumar, Jacob, Cutrer, Link, Atkinson, Wetjen. Drafting the article: all authors. Critically revising the article: Kumar, Welker, Cutrer, Link, Atkinson, Wetjen. Reviewed submitted version of manuscript: Welker, Cutrer, Link, Atkinson, Wetjen.

\section{Supplemental Information}

Previous Presentation

A portion of this study was presented at the Winter Clinics for Cranial and Spinal Surgery in Snowmass Village, Colorado, on February 26, 2014.

\section{Correspondence}

Ravi Kumar, Department of Neurosurgery, Mayo Clinic, 200 1st St. SW, Rochester, MN 55905. email: kumar.ravi@mayo.edu. 